Received 00th January 20xx, Accepted 00th January 20xx DOI: $10.1039 / \times 0 \times x 00000 x$

\title{
Modelling biocompatible ionic liquids based on organic acids, and amino acids: challenges for computational models and future perspectives.
}

\author{
Enrico Bodo, *a
}

\begin{abstract}
In this short review I shall highlight the basic principle and the difficulties that arise in attempting the computational modeling of seemingly symple systems which hide an unexpected complexity. The target of this review are biocompatible ionic liquids which are based on the coupling of organic or amino acids anions with metabolic cations such as cholinium. These substances have been the subject of intense research activities in the last years and have attracted the attention of computational chemists. I shall show that the computational description of these substances is far from being trivial and requires the use of sophisticated techniques in order to account for a surprisingly rich chemistry that is due to several phenomena such as polarization, charge transfer, proton transfer equilibria and tautomerization reactions.
\end{abstract}

\section{Introduction}

Computational modelling of complex amorphous and heterogenous systems is a challenging, and sometime frustrating, task for chemists. Nevertheless, the possibility of providing reliable predictions using models and computations represents a huge advantage in terms of designing more efficient research activities and to replace tedious and expensive screening processes in the laboratory. Unfortunately, even the most sophisticated state-of-the-art computational techniques, are still far from being reliable enough when the complexity of the systems under investigation increases.

When dealing with system of small sizes (typically isolated molecules or small aggregates) extremely accurate methods are now available to chemists. Some of them are based on the calculation of utmost complicated electronic wavefunctions as in multireference ${ }^{1}$ or coupled cluster ${ }^{2}$ methods, while others rely on electronic densities (Density Functional Theory, DFT) and the concoction of elaborate and specific-purpose energy functionals. 3,4 These methods allow the computations of molecular properties to an unprecedented accuracy with the additional appeal of being readily available while the corresponding measurements are sometimes plagued by practical difficulties.

The extension of these first-principle, accurate methods to large complex systems such as those typically encountered in material chemistry is not possible, at least in a straightforward way. On the one hand, performance issue limits their practical applicability, on the other, the fine-grained information they provide is often unneeded and their use is hampered by a

a. Chemistry Department, University of Rome "La Sapienza", P. A. Moro 5, 00185 Rome, Italy.Email: enrico.bodo@uniroma1.it negative trade-off between their cost and the usefulness of the outcomes.

A typical example is the solvation of molecules. Since one cannot extend the accurate methods to the entire solute+solvent system, either one lowers the calculation accuracy of both or has to privilege one of the two (typically the solute) and adopt a very rough model for the other (solvent). 5,6 Another crucial issue is dynamic. The aforementioned methods are "static", i.e. they provide an instantaneous "snapshot" of a given chemical system, but yield only limited information about its chemical evolution. The problem is that, very often, the chemistry of a given complex material stems from emerging properties which do not simply come from those of the constituents 7,8 . These emerging properties surface from complex dynamical effects due to the way in which the molecular components of the material behave collectively on a certain timescale.

In typical chemistry contexts, "dynamics" has the implicit meaning of "motion of the atoms". The issue here is that, from the physical standpoint, and apart from trivial cases, the "atom" in a molecular system is a somewhat ambiguous and elusive concept. The constituents of matter at a fundamental level are electrons and nuclei, not atoms. Both of them are quantum particles whose behaviour is governed by the Schrödinger equation. The cornerstone of the chemical interpretation lies in the possibility of shifting from a language based on nuclei and electrons to one based on atoms (the language of chemistry). This is precisely what can be done by applying the BornOppenheimer (BO) approximation. The general approach consists in separating the molecular Schrödinger equation into an electronic equation (often solved using the techniques mentioned above) and a set of coupled nuclear equations. The BO approximation allows to decouple the set of nuclear equations, thus assuming that the nuclear motion on a given electronic state is independent of all the others. More 
importantly, the BO approximation let us describe the motion of the nuclei in a given electronic state as governed by a potential that is the electronic energy. When the BO approximation holds, nuclei can be "blended" into atoms and the electrons can be seen as the source of the interatomic potentials (hence the forces) acting on them.

At difference from electronic calculations, computational chemists do not have access to similarly accurate quantum methods to treat the nuclear or atomic motion, except for simple few-atom systems where, anyway, the full quantum treatment of nuclear motion reaches an extreme level of complexity. ${ }^{9}$ In most cases, the motion of the heavy particles (nuclei or atoms) is described approximating the quantum dynamics (the nuclear Schrödinger equation) with a classical one (Newton's law). Conventionally, this approximation goes under the name of molecular dynamics (MD).

Neglecting the quantum nature of the nuclei leads to a series of systematic and non-systematic errors consisting in overlooking zero-point-energies and tunnelling, neglecting coherence, symmetry and exchange effects and ignoring the discrete nature of the energies of the molecular vibrations. Even though many of these effects can actually be accounted for in various ways, 10 they have yet to become typical addition to traditional MD techniques.

Overlooking quantum nuclear motions is not the only issue affecting typical MD simulations. Another crucial point is the accuracy of the forces (or of the potentials) that govern the classical motion of the particles. Here lies the distinction between the several flavours of MD: either one computes the forces using the gradient of the electronic energy as it stems from the electronic Schrodinger equation, or one has to model the forces using a reasonable, but simple, parametrization known as the "force field" 11 . The former gives rise to the approach known as Born-Oppenheimer MD (BOMD) also called ab-initio MD (AIMD). ${ }^{12}$ The latter consists in the so called "classical" MD. ${ }^{13}$ The choice is dictated by the nature and timescales of the events one has to sample and by the number of atoms that are necessary to describe them. AIMD is often limited to hundreds of ps and few thousands of atoms, while classical MD is able to reach the ms regime and describe the motion of millions of atoms. The huge gap in between can be filled by methods that require a less expensive way to solve the electronic Schrodinger equation such as semiempirical approaches. ${ }^{14,15}$

Ionic liquids (ILs) are a broad class of materials that has shown an exceptional versatility and whose potential applications range from electrochemistry to medicine, from industrial process to biomass processing agents. ${ }^{16,17}$ Since their inception in the research field 18 ILs have been sometimes considered inherently "green" solvents, but recent research has shown that they are actually toxic toward organisms and less environmentally-benign than previously assumed ${ }^{19-24}$

Once realized that ILs are less green than expected, part of the research has steered toward the quest for truly biocompatible ILs. One of the earliest examples of such biocompatible ionic compounds 25 consisted in the coupling of choline chloride with organic, naturally occurring acids. Since then, it has become clear that the cholinium cation, a metabolic harmless substance, could replace of the imidazolium ions while the deprotonated forms of naturally occurring organic acids could be used instead of fluorinated anions such as $\mathrm{Tf}_{2} \mathrm{~N}$ of $\mathrm{SF}_{6} .{ }^{26}$ Further improvements can be obtained with the use of renewable and readily available compounds such as naturally occurring amino acids. ${ }^{27}$ These biocompatible ILs have the potential to be exploited as truly green solvents from their synthesis to their use. ${ }^{28,29}$

Most of biocompatible ILs are members of the class of ILs known as "protic ionic liquids" (PILs) that are characterized by the presence of a pervasive hydrogen bond network due to the presence of protons and acceptor/donor sites. PILs have been shown to be promising candidates for potential applications in medicine ${ }^{30,31}$ and biomass processing. ${ }^{32}$ One of the key features of PILs is the possible mobility of protons which may act as faster charge carriers than the ions themselves and allow these substances to find useful applications in electrochemistry. ${ }^{33}$

The structure of ILs at the nanoscopic and mesoscopic scales has been thoroughly summarized in recent literature. ${ }^{17,34-39}$ ILs show a complicated hierarchy of structures depending on their molecular components: the presence of long alkyl chains induce the appearance of self-assembled nano-structures, 40 the presence of acceptor/donor sites spawn complicated hydrogen bond structures, ${ }^{41}$ unconventional aggregation phenomena like same-charge clustering has also been detected. ${ }^{42,43}$

This broad variety of structural phenomena represents a challenge for computational chemists and has resulted in the implementation of an array of different computational techniques ranging from ab-initio molecular calculations at the nanoscopic scale, ${ }^{41}$ to simulation protocols based on MD well into the meso-scale. ${ }^{44}$

Here we shall focus on the computational challenges that lie in the study of biocompatible ILs i.e. those liquids made using anions such as organic acids (AILs) or amino acids (AAILs), and the cholinium cation (Ch). ${ }^{45,46}$

Since their early synthesis, ${ }^{47-49}$ AAILs have represented a viable biocompatible replacement for traditional ILs in various applications: lubricants $50,51, \mathrm{CO}_{2}$ absorbing media ${ }^{52-56}$, protein extraction media ${ }^{57-61}$, and biomass processing agents ${ }^{62-64}$.

Soon after initial synthetic works, early computational studies 65,66 have appeared. The essential result was that the binding motifs in the isolated ionic pairs turned out to be very similar along the entire AA anions series. This result did not explain the huge variation in the bulk properties of the fluids which were strongly dependent on the peculiar amino acid anion. ${ }^{67}$ In a second moment, additional studies based on classical MD simulations and X-ray diffraction patterns ${ }^{68-70}$ showed the emergence of long range aggregation patterns, but a simple correlation between their bulk properties and the nature of the molecular components still eluded researchers. These liquids represent the ideal playground for theoretical methods because, as it has emerged, they possess a unique and complicate structure which defy a simple a straightforward computational characterization. 


\section{Methods: state-of-the-art}

Simulations of ILs are carried out by using a variety of methods. These range from accurate quantum chemistry calculations on isolated molecules and dimers to MD simulations comprising $\sim 10^{6}$ atoms. It is not our intention to summarize them all here and we shall limit ourselves to a brief survey of the methods based on first principles (electrons and nuclei) and of those which retain an atomistic approach. Non-atomistic simulations such as coarse grained MD ${ }^{71,72}$ and continuum models 73 are also possible, but we defer the reader to the specialized literature for these.

The simulation protocols and methods can be simply organized in a hierarchical way such as that of Figure 1. Going from isolated molecules to the bulk fluid, the typical simulation shifts from ab-initio methods (wavefunction- and DFT-based) toward approaches such as classical (force field-based) MD. It is worth point out that this is a hierarchy based exclusively in terms of system size, but it does not necessarily reflect the accuracy of the methods involved. For example, classical MD can perform much better in matching experiments than ab-initio based MD depending on how well the force field has been parametrized. Between the isolated molecule/dimer systems and the bulk simulations, there is an intermediate level where isolated, small aggregates of ions are investigated. This level is only seldom used but represents the ideal playground for gradually switching from the isolated molecules to the bulk phase and looking at how molecular properties are gradually affected by an increasing presence of a surrounding environment. The advantage of using relatively small, finite-size aggregates (clusters) is that the highly accurate calculations typically used for isolated molecules are still doable and that the sampling of their geometries and of other possible dynamical phenomena through MD is available as well. ${ }^{74}$ The main drawback is that clusters do have a finite size and border effects are important. When moving to the bulk phase, simulations are normally carried out using MD, which means that one uses a classical approximation for the nuclear/atomic motion. In the simulations of the bulk phase, the border presence is effectively removed by imposing an artificial translational symmetry in three dimensions so that replicas of the molecules contained in a reference (cubic) cell are implicitly taken into account when the potential is evaluated near the cell border. In this way, all the molecules inside the reference cell are always surrounded by other molecules.

In AIMD the forces governing the motions of the nuclei are calculated via some kind of approximate resolution of the electronic Schrödinger equation (typically DFT) by differentiating the resulting electronic energy. The chemical topology in AIMD simulations is not fixed but stems naturally from the electronic density as it arises from first principles (hence the "ab-initio" in the name). In other words, the atoms are free to change either their coordination number or their valence depending on the immediate surroundings.

In classical (force field-based) MD the nuclei are blended into "atoms" and the interatomic potential consists in a set of simple parametric expressions whose variables are angles and distances. Each atom is assigned a fixed partial charge, a volume (through the use of short-range repulsive potentials), and a long-range van der Waals attractive potential. The groups of atoms that are covalently bound have a fixed coordination scheme. In classical MD the atoms are not free to change their valence and bonding patterns. In other words, in classical MD the chemical topology is fixed a-priori and does not change during the temporal evolution. This last limitation can be overcome using reactive force fields, which allow for changes in coordination number, but have only seldom been applied to ILs. 75
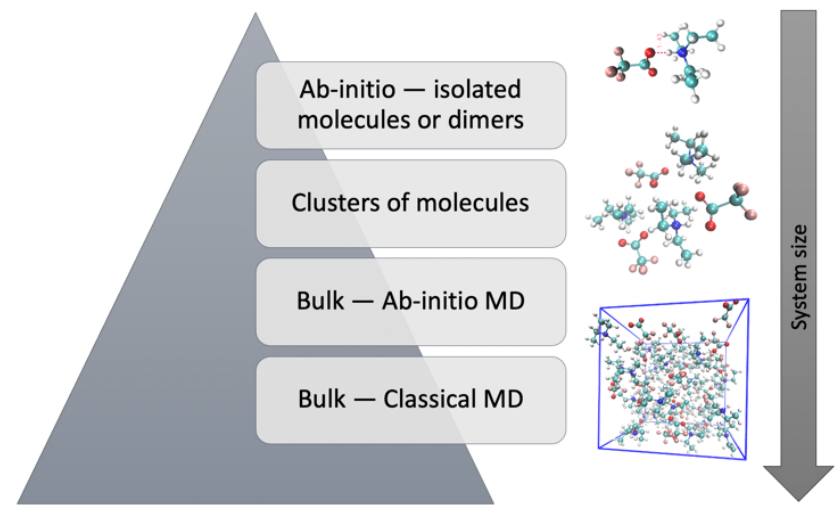

Figure 1: Scheme of the simulation paradigms based on atomistic descriptions.

\section{Static ab-initio calculations}

Ab-initio calculations of isolated molecules or of interacting dimers have a twofold purpose. On the one hand, they provide a description for the pair interactions that are part of the fluid cohesive energy and determine its short-range structure. On the other, they can be used as benchmarks and a source of data for the parametrization of force fields.

The main advantage of performing a calculation on isolated ionic couples is that a great accuracy in geometries, energies and electronic densities can be had with a modest computational effort. Very sophisticated analysis of the resulting wavefunctions (or molecular orbitals) such as atoms in molecules (AIM) ${ }^{41}$ and fragment molecular orbitals (FMO) 76 can be then exploited to quantify effects such as polarization and charge transfer and to extract realistic intermolecular potentials.

For example, the analysis of the wavefunction in a hydrogenbonded ionic pair can provide information about the strength and nature of the hydrogen bond. As an example, we report the results of a DFT calculations on the ionic couple of triethylammonium mesylate (TEAMs). A mathematical procedure called orbital localization 77 can be used to identify and visualize the molecular orbitals which are involved in the hydrogen bond between the oxygen and the ammonium group which we report in Figure 2. This kind of approach is useful not only because it gives information about the bonding motifs between anions and cations, but also because it provides the necessary data to build interaction models for the bulk phase. More information and examples on this topic can be found in ref. ${ }^{41}$. 
Static ab-initio calculations can be extended easily to larger aggregates (clusters) retaining the same level of accuracy, albeit at an increased computational cost. Having the possibility of moving into a realm that is at the border between the isolated ionic pair and the bulk phase allows computational chemists to predict accurately bulk properties such as vibrational absorption spectra. ${ }^{78,79}$ The typical size of an IL cluster is large enough to make wavefunction-based techniques unaffordable, hence computationally cheaper methods based on DFT are dominant in this context. The choice of the calculation method (i.e., the functional and the basis set) is crucial in order to achieve a given accuracy within an acceptable cost. General prescriptions, based on functional/basis accuracies, can be obtained by extensive testing 80 and by comparison with experimental observables such as vibrational spectra. ${ }^{81}$

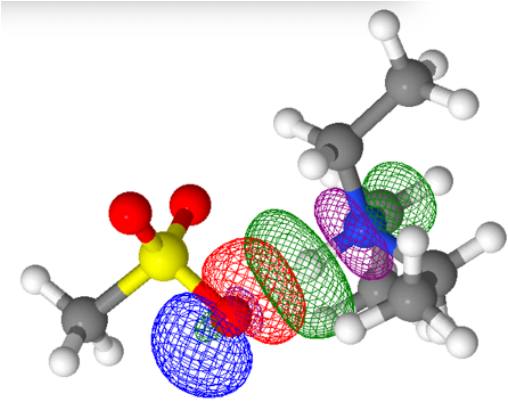

Figure 2: The two molecular orbitals involved in the $\mathrm{H}$-bonding feature between a mesylate anion and a triethylammonium cation. The red/blue one is a lone pair on the oxygen, the green/purple one is an anti-bonding orbital localized on the $\mathrm{N}-\mathrm{H}$ bond.

\section{Force field-based molecular dynamics}

The vast majority of computational studies on ILs have been carried out using classical MD. This is the method of choice for the investigation of molecular liquids and the recent development in the field have been summarized in several reviews. ${ }^{74,82-85}$

Classical MD relies on the parametrization of the interatomic interactions and its results are obviously dependent on the quality of the latter. Interatomic interactions are written in terms of simple analytical expressions which are typically divided into two parts: a many body expression for the shortrange potential used to preserve the topology of the covalently bound entities (up to 4-bodies) and a sum of two-body potentials for modelling the long-range, anisotropic intra- and inter-molecular interactions. Such a simplified expression of the interatomic potential completely bypasses the need to evaluate forces using explicit electrons and results in a performance gain of several orders of magnitude with respect to ab-initio methods.

One of the strengths of classical MD is the ability to predict the structure of the fluid and to interpret structural measurements such as diffraction data. In particular, many ILs show the existence of peculiar and transient long-range structural organization. These transient structures reveal themselves in $\mathrm{X}$ ray diffraction patterns through the appearance of low- $Q$ scattering peaks whose direct space reciprocal value put their sizes in the nanometric range. ${ }^{40,86-88}$ This long range order has been the subject of numerous studies based on classical MD which aimed at characterising the shape and nature of the structures involved. $68,70,89$

While the force field-based simulations have shown an outstanding precision in replicating and illustrating the structure of several ILs they have had a much lower success rate in reproducing their dynamical properties. Here is where polarization issues arise. The great majority of existing force fields optimized for ILs do not include polarization. In other words, the atomic charges are fixed and do not change depending on the surrounding. In addition, if the charge of the ions is set to match \pm 1 , this approach greatly overestimates the friction inside the fluid leading to erroneous prediction of properties such as viscosity or conductivity (see the discussion on the charge transfer problem below). An often used workaround consists in scaling the ionic charges by a constant factor (often determined a priori, using electronic structure calculations) to reduce the strength of ionic interactions and the fluid friction. ${ }^{90,91}$

\section{The polarization and charge transfer problems}

Polarization is simply connected to the distortion of the molecular and atomic electronic density due to the presence of a nearby charge. The polarization of the molecular electron density induces the formation of an additional dipole moment whose interaction with the charge is attractive and proportional to the polarizability of the molecule. This interaction between the charge and the induced dipole has a shorter range than electrostatic ones but nevertheless represents an important component of the total energy in ILs. Beside this interaction which can be rationalized as a two-body potential, polarization manifests itself also through many-body effects: the induced dipoles are not isolated, but they interact with each other giving rise to attractive or repulsive interactions depending on their mutual orientation. In ILs these many-body interactions between the induced dipoles generally reduce the overall electrostatic cohesive energy, thus explaining why the force field models that include polarization effects reduce friction and results in a more reliable calculations of dynamical quantities.

An issue closely connected to polarization is the so-called "charge transfer" problem. Broadly speaking one can see the effect of charge transfer by noting that, in ILs, the overall charges of anions and cations are less than unity when proper calculations techniques are applied (AIMD for example). ${ }^{92} \mathrm{As}$ an example, in Figure 3, we report the distribution of ionic charges obtained in a 10 ps simulation of TEAMs (see above). Since this simulation is based on the evaluation of the electronic density, all many body effects (polarization and charge delocalization) are included. Despite TEAMs being a system with small ions and weak ionic pairing, we have found an overall reduction of the total ion charge from $5 \%$ to $10 \%$. In other systems where the charge is delocalized over larger molecular structures and where ionic pairing is stronger, the net ionic charge can be as low as \pm 0.75 .93$

Both polarization and charge transfer are a quantum effects due to the distortion and to the migration of electron density between the ions. Because of this, both phenomena are 
naturally accounted for by simulations that compute the true electronic density from a solution of the electronic Schrodinger equation.

These effects are not included in classical MD simulations that use fixed atomic charges. There exist several techniques that can be used to account for polarization effects in classical $\mathrm{MD}, 91,94,95$ but they generally increase the computational cost of the simulations. As we have mentioned, introducing polarization is crucial because it reduces the electrostatic overbinding (due to fixed charges) and aligns the calculated frictional properties with the measured ones. in other words, polarization is often necessary to achieve a correct computational representation of the dynamics in ILs.

On the other hand, the issue related to charge transfer is particularly difficult to be included in a reliable way using classical MD simulations where the net charge of the ions is necessarily fixed and not allowed to fluctuate as it does in reality (see Figure 3). As we mentioned above, reducing the overall ionic charges by uniform scaling or introducing a dielectric screening are known and broadly used workarounds. 90

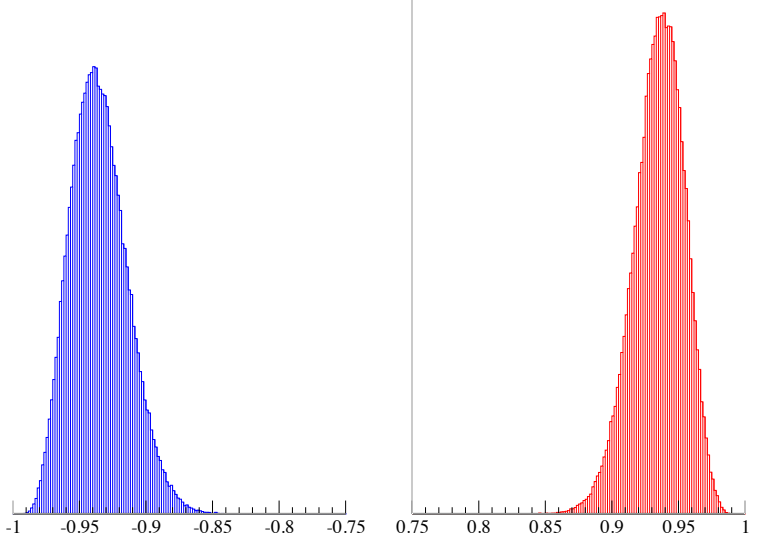

Figure 3: Distribution of computed total ionic charges in the TEAMs liquid for the mesylate anion (left) and triethylammonium cation (right) along $10 \mathrm{ps}$ of simulations.

\section{Ab initio molecular dynamics}

The term ab-initio MD (or Born-Oppenheimer MD) 12,96,97 encompasses all techniques that rely on the evaluation of the electronic energy and of its gradient from the electronic Schrödinger equation. These simulations have, in principle, several advantages over classical ones:

- They naturally include polarization effects;

- They include many-body effects both at short and at long range;

- Fluctuating atomic charges can be derived from the occupation numbers of suitably localized orbitals; 98

- Anharmonic motions are accounted for, which, together with the computation of time dependent dipole moments, leads to a simple procedure to generate vibrational absorption spectra;

- Chemical reactions, isomerization or tautomerization reaction are free to take place.

Despite seemingly a remedy for the several drawbacks of the force field-based techniques, AIMD is far from being some kind of accurate "black box" that yields certainly valid results. First of all, there are "hidden" assumptions: (i) that the molecules evolve on a single potential energy surface (Born-Oppenheimer approximation), and (ii) that the nuclear quantum effects are small enough so that we can ignore them and treat the atoms as classical objects obeying Newton's equations of motion.

In addition, the way in which the electronic Schrodinger equation is solved does matter and not all techniques can claim a universal accuracy. Since computational cost is a decisive issue for AIMD, the choice for solving the electronic equation normally falls on the DFT method. As a result, the outcomes of a given simulation are obviously dependent on the chosen functional form. For example, simple and widespread functionals such as PBE and BLYP often underestimate reaction barriers 99 leading to incorrect kinetic in the simulation.

Despite problems related to functional choice, in the absence of chemical reactions, the fluid dynamics as captured by simulations based on ab-initio calculations has been proven to be very accurate. For example, AIMD represents a unique opportunity to study intrinsically inhomogeneous situations such as the evaporation process of ILs where the inevitable border effects have to be correctly accounted for by explicit evaluation of the electronic densities. 100,101

Another important progress in incorporating AIMD into the arsenal of techniques available to the chemists consists in its use as a source of high-quality reference data from which classical force fields can be obtained and optimized. 102,103

All the aforementioned positive features of AIMD come with a price that corresponds to an excessive usage of computational resources that seriously limits its applicability in describing longtimescales phenomena. Such an increase in the computational costs generates two drawbacks: both the time span of the simulations and the sizes of the systems which are amenable to be treated with AIMD are dramatically limited. A quite extensive AIMD simulation could reach hundreds of picoseconds for a system consisting of about a thousand of atoms.

\section{Middle-of-the-road: semiempirical approaches}

It is quite obvious that the aforementioned increase in computational needs depends on the fact that AIMD has to provide somehow an approximate solution of the electronic Schrödinger equation at each step of the time-propagator. It therefore follows that any possible mean of reducing the requirements of the time-consuming electronic problem would also reduce the computational cost, hence allowing the exploration of longer timescales and larger systems. A possible way to do this is to employ a semi-empirical method, known as Density Functional Tight Binding (DFTB), 104,105 which combines a good accuracy of the electronic energies at a reduced computational cost. DFTB is based on a second-order expansion of the Kohn-Sham total energy with respect to charge density fluctuations. The latest version of DFTB includes the extensions of this energy up to the third-order that is crucial for an accurate representation of hydrogen bonding. 106 In DFTB, the electron density is written as a reference density (derived from DFT methods and stored in the code as a parameter) plus a perturbation which include the effect of the atom-atom repulsive term (mainly at short range), of a "second order" term 
that allows the charges to fluctuate (self-consistent charges DFTB or SCC-DFTB), and of a "third order" term (DFTB3 method) which represents a further modification of the Coulomb repulsion between atoms.

A DFTB calculation relies on an "a priori" knowledge of the many parameters appearing in the energy expression. These parameters are contained in the so-called "Slater-Koster" files. There are different sets of parameters available, many of which have been tested and validated for specific applications.

The DFTB simulations typically used for ILs modelling are based on the 3ob set 107 of parameters and a third-order expansion of the energy (DFTB3). In this variant, the DFTB method has been successfully applied to a large number of chemical systems ranging from metallic nanoparticles to ionic liquids $108-110$ and it has been shown to reach comparable accuracy to DFT methods.

\section{Computational challenges}

\section{The problem of long timescale}

From the point of view of MD techniques, one of the difficulties that one encounters with ILs in general, and AAILs in particular is dealing with the high viscosity of the fluids. Viscosities are several orders of magnitude larger than water, ranging from $10^{2}$ to $10^{5} \mathrm{mPa} \cdot \mathrm{s}$. In terms of computations, this translates into the need to use very long simulation times. In other words, the time needed to see a change inside the structure of the simulated liquid often becomes so long that it requires the use of simplified and less accurate computational strategies. A pictorial example of the sluggishness of this kind of fluids is reported in Figure 4. We have simulated TEAMs (a fluid with a viscosity of about $100 \mathrm{mPa}$ s) for 400 ps using the DFTB semiempirical method. The cation centres of mass are shown as blue spheres, the anion ones in red. We have plotted a sphere each $30 \mathrm{fs}$ of simulation time.

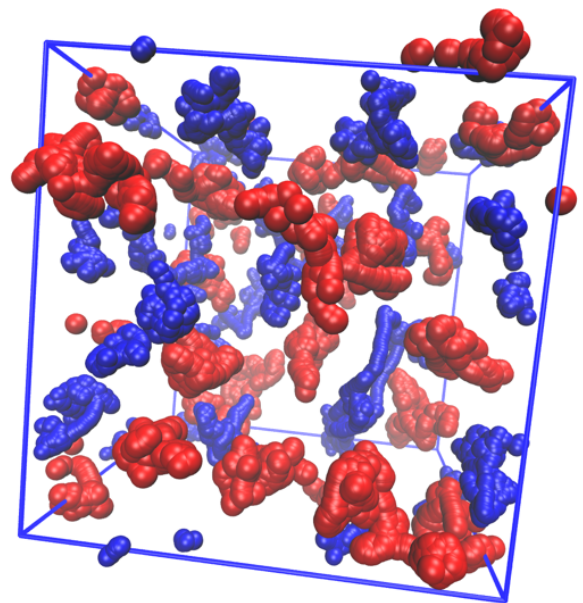

Figure 4: Temporal evolution of the cation and anion centres of mass during 400 ps in triethylammonium mesylate.

The movement of the ions in roughly half a ns is extremely limited and most of them appear to simply oscillate randomly around the initial positions. Only few of them show an appreciable drift or exchange position over time. Such example should clearly point out how, for ILs, the computational observation of several dynamical events (ionic diffusion, ligand exchange, rearrangements of solvation shells etc.) requires extreme simulation times that often lie beyond the edge of current possibilities especially if accurate calculations of the forces are involved. In other words, the more accurate is the modelling of the interactions, the less likely is that one is able to sample times long enough to provide converged thermodynamical quantities.

\section{The problem of ionicity}

While slow dynamics and polarization effects are issues common to all ILs simulations, ionicity is an issue that affects mainly protic ionic liquids (PILs). Regardless of their actual synthetic procedure, PILs can be thought as stemming from a simple acid/base reaction:

$\mathrm{AH}+\mathrm{B} \rightarrow \mathrm{A}^{-}+\mathrm{BH}^{+}$

where, in order to form a true PIL, the ensuing product has to be a fully ionized liquid. In other words, the proton transfer from $A$ to $B$ must be quantitative and all neutral components (the reagents) must disappear.

From basic chemistry, it is clear that reaction (1) is an equilibrium process that can be more or less shifted to the right depending on the propensity of the acid/base pair to share the proton. Unfortunately, in non-aqueous systems, a simple relation between the equilibrium position and the nature of the molecules seems to have eluded the research community.

One of the most obvious indicators of the propensity for proton release and capture is the pKa. For PILs it is customary to relate the extent of reaction (1) to the difference in the pKa of $\mathrm{AH}$ and $\mathrm{BH}^{+}, 111,112$ but it turns out that, with the exception of very large $\Delta$ pKa, 113 the ultimate fate of reaction (1) is very difficult to predict. A large $\Delta p K a$ leads to highly ionized liquids, a small one leads to a partially neutral system with the possible occurrence of phase separation of the volatile neutral components. ${ }^{114-116}$ In all intermediate cases, however, the metric based on pKa is not conclusive as one can realize by observing the different ionicity degrees obtained by mixing acetic acid and different amines with similar pKa. ${ }^{117}$ Even in homologous series of PILs, ionicity (hence viscosities and conductivities which are strictly related to it) do not show a clear relation with pKa, 118 but rather with the ability of the ensuing fluid to (self-)solvate the ions, 119 with the proton affinities of the bases, 120 and with other thermodynamics factors such as the entropy change associated with reaction (1). ${ }^{121}$ That the self-solvation properties of the final liquid towards the ions is a crucial, albeit difficult to predict, ingredient to understand the extent of equilibrium in reaction (1) has clearly emerged in a series of recent works. ${ }^{122-125}$ An example of how simple molecular indicators can be misleading in understanding ionicity in a given PILs is triethylammonium acetate (TEAAC). Judging from $\mathrm{pKa}$, one expects to find a majority of acetates and ammonium cations. In a recent work, backed up by experimental evidence, we have shown that this is not the case. ${ }^{114,115}$ In its pure form, TEAAc is a liquid composed by roughly two thirds of neutral molecules which solvate the remaining third of the ions. A pictorial way to represent this situation is reported in Figure 5 . This figure shows 
the data obtained using a DFTB-based MD simulation and depicts the volumetric positions of the ionic (red) and neutral (blue) components of the fluid obtained averaging over $300 \mathrm{ps}$. The proportion of ionized and neutral phases stems naturally from the simulation protocol that does not enforce an a-priori chemical topology and allows for proton transfer to occur as driven by the gradient of the electronic energy.

The problem of not having a unique, well-defined ionic composition (at least without an a-priori knowledge) is a serious one and assumes the form of a critical conundrum. While on the one hand it is desirable to employ a method with a variable chemical topology able to describe the proton transfer equilibria (AIMD), on the other, to reach the necessary simulation times and to achieve a reliable description of thermodynamic equilibrium in these viscous systems, one should resort to the fast force-field based methods which however do normally rely on a fixed topology. In other words, if the position of equilibrium (1) is not known or uncertain, the possibilities of obtaining a clear-cut answer from simulations are rather limited. Useful indications can certainly be had from the accurate computations of proton transfer energy profiles in isolated pairs, 126,127 and from AIMD simulations, ${ }^{128-130}$ but the reliable prediction of equilibrium (1) in condensed system still defies a straightforward computational approach.

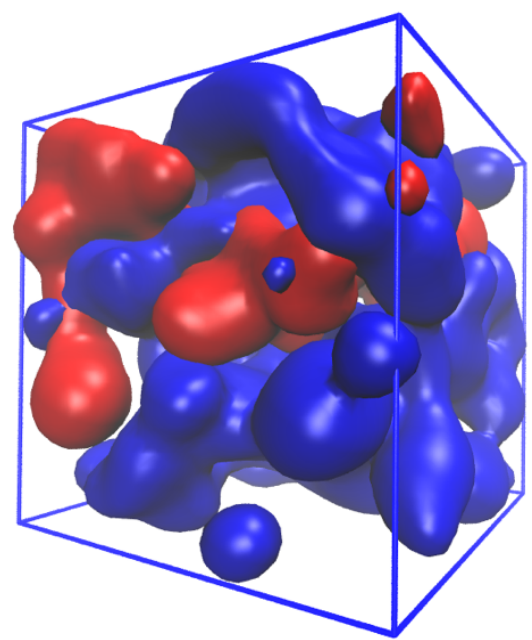

Figure 5: Average volumetric positions of triethylamine and acetic acid (blue) vs those of triethylammonium and acetate ions (red).

\section{Like charge aggregation phenomena}

Since earliest computational studies, it was apparent that the structure of PILs in general, and AAILs in particular, was not as simple as initially thought. For example, the presence of low- $Q$ scattering peaks in the $\mathrm{X}$-ray diffraction patterns indicated that there existed some form of structural aggregation at the nanoscale that calculations on isolated dimers were unable to grasp. ${ }^{68,69}$

From some of our recent studies, ${ }^{129,131}$ it was clear that the structure of these fluids was determined, not only by the interactions between molecular components of opposite charges, but also by those between likely charged ones. Modeling such balance requires necessarily a great accuracy in the calculation of the interatomic potentials because of the need to properly account for polarization phenomena. Hence, computational studies based on an ab-initio evaluation of the electronic energy (AIMD) played a crucial role in understanding their structure.
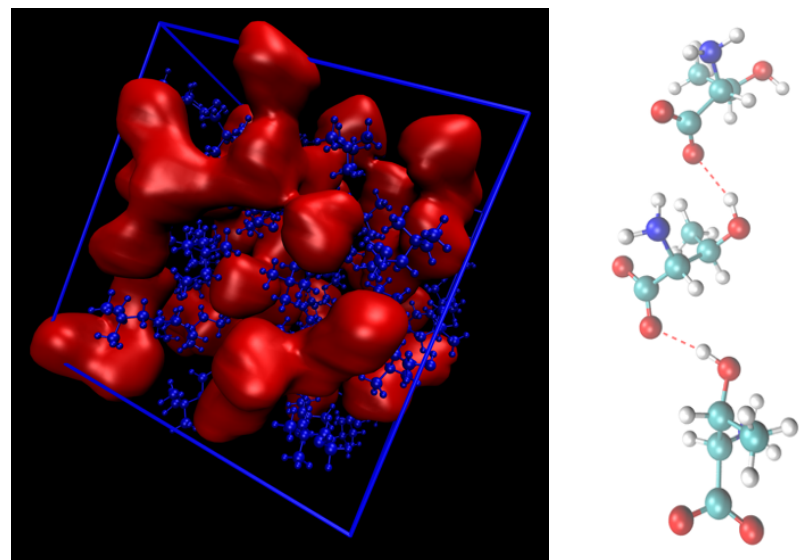

Figure 6: Snapshot of a AIMD simulations containing [Ch][Thr]. The anionic moieties are represented by the red iso-surfaces, while the cations correspond to the blue structures. The formation of oligomers of anions is evident as well as the nano-segregation between the oppositely charged moieties. Right: examples of hydrogen-bonded clusters of [Thr] anions as extracted from the simulations.

As suggested by a series of works by Ludwig and coworkers ${ }^{132-}$ 135 the propensity of like-charge ions to aggregate could be a key factor in ILs where $\mathrm{H}$-bonding interactions are important and strong enough to overcome electrostatic repulsion. Like-charge aggregation is also favored by charge delocalization effects (for example carboxylates or sulphonates), by charge transfer through hydrogen bonds which reduce the overall ionic charge of the ions and by the weakening of electrostatic interaction due to the surrounding dielectric response of the medium (polarization).

The extent to which like-charge interactions exist and influence the frictional properties of AAILs has been explored by us and we have found that, even with the simplest aliphatic amino acids, the results of AIMD simulations suggest conspicuous anion-anion pairing through $\mathrm{H}$-bonding between the aminoand carboxylate groups $43,129,136$. Interestingly, the anion-anion binding energies show an evident correlation with the measured viscosities of the corresponding liquids. A pictorial example of these aggregated anionic domains as emerges from AIMD simulations is presented in Figure 6 where the aggregation of the [Thr] $]^{-}$anions is highlighted using a redcolored volumetric density. An example of a trimeric $\left[\mathrm{Thr}_{3}\right]^{3-}$ anionic structures is also shown.

Anion-anion aggregation phenomena in AAILs recently emerged also from neutron diffraction data ${ }^{137}$ of the [Ch][Phe] liquid that confirm the presence of anion-rich domains supported by interanion hydrogen bonds. Interestingly, the persistence of these anionic dominated region in the fluid might also be responsible for certain low-Q correlations spotted in SAXS diffraction patterns which are otherwise difficult to attribute in the absence of apolar aliphatic chains. ${ }^{138}$ 


\section{The problem of tautomers}

Most biocompatible ionic liquids, at the moment, are derived from amino acids anions and from cholinium cations. Since the cholinium cation is a very poor base, they are generally considered to be fully ionic substances. In other words, when applied to an amino acid/cholinium pair, equilibrium (1) is completely shifted to the right. However, recent calculations by us 128,139 have shown that the aforementioned anionic domains can host a rather complex proton chemistry due to additional tautomerization equilibrium processes.

We have shown that the amino acid anions, when they contain an additional protic function such as $-\mathrm{SH},-\mathrm{PO}_{3} \mathrm{H}_{2}$ or $-\mathrm{COOH}$, can tautomerize and form a zwitterionic-anionic structure where the additional proton has moved onto the $-\mathrm{NH}_{2}$ group. The mechanism of two possible tautomerization pathways in the anionic moiety of AA anions with protic side chains is reported in Scheme 1.

The presence of multiple partial charges on the zwitterionic anion and the spawning of new acceptor donor sites further weakens the overall anion-anion electrostatic repulsion and allows for an even tighter anion aggregation phenomena. ${ }^{140}$ The resulting zwitterionic dimers for [Asp] and [Glu], shown in Figure 7 , are particularly stable with interaction energies which are comparable to those of the cation/anion counterpart. ${ }^{43}$

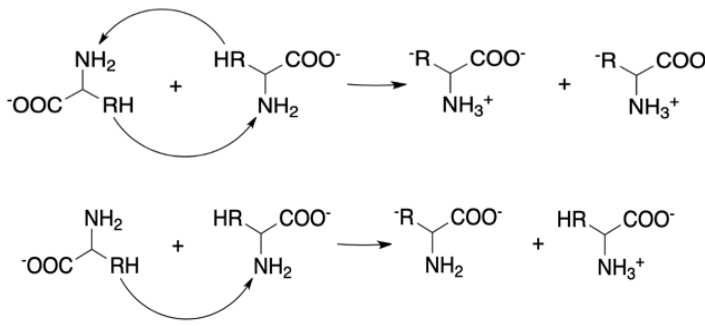

Scheme 1: Possible tautomerization reactions in AA anions with protic side chains. On top we report the formation of two zwitterionic anions; below, the formation of a dianion and a neutral specie. Here $\mathrm{RH}$ is a typical protic side chain group such as $\mathrm{COOH}, \mathrm{PO}_{3} \mathrm{H}_{2}$ and $\mathrm{SH}$.

It is clear at this point that, these additional proton transfer equilibria pose an additional problem in the computational description of the fluids. The extent of the tautomerization reaction is not easily predictable and also difficult to ascertain from experimental data. Classical, force field-based MD cannot be easily applied because the composition (zwitterionic vs anionic species) at equilibrium might not be known in advance, hence the charge states of the functional groups is uncertain. AIMD would indeed take care of this problem, but the time scales required for obtaining a fully equilibrated sample would render these simulations extremely unpractical.

\section{Conclusions}

Protic ILs have been, since their inception in the landscape of ionic media, important candidates for replacing, especially in their biocompatible formulations, traditional ionic liquids. They have already been at the center of an intense activity in fields such as biomedical research and biomass treatment. From the fundamental point of view, the computational description of these liquids and, eventually, of their interaction with biomolecules represents a challenge for the near future.
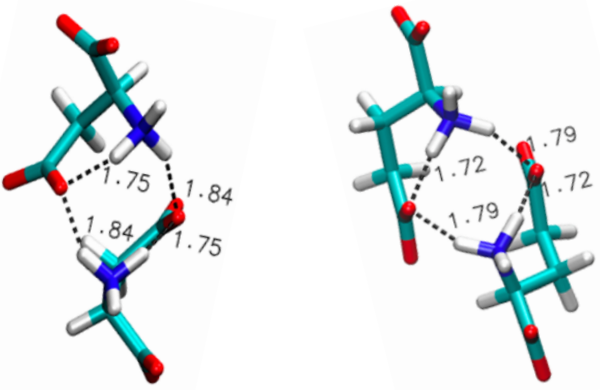

Figure 7: Optimized structure of the zwitterionic dimers $[\mathrm{Asp}]_{2^{2-}}(\mathrm{left})$ and $[\mathrm{Glu}]_{2^{2-}}$ (right).

The problem with these substances is that their degree of ionicity, their ability to transport charge, the possible phenomena involving proton transfer are very dependent upon the tendency of the involved molecular species to bind the proton which, in turn, stems from a complex combination of diverse effects. Charge migration, polarization, many body effects, unusual (hydrogen-bond driven) aggregation phenomena, tautomerization reactions and other proton transfer processes, all contribute to draw a peculiarly complex landscape which defies the more traditional approaches of computational chemistry. Simple a-priori indicators such as proton affinities and $\mathrm{pK}_{\mathrm{a}}$ do not show a clear and simple correlation with crucial characteristic of the fluid as ionization degree, frictional properties and tautomeric abundances. In addition, the presence of unusual tautomeric ionized states in protic amino acid anions brings further complications to the overall picture.

For those systems based on simple (aliphatic and aromatic) amino acids coupled to very poor bases such as cholinium, an accurate treatment of polarization within force field-based methods should be sufficient to achieve a very good accuracy. An accuracy that could even be increased by introducing an especially suited uniform scaling of the ionic charges to account for charge transfer. The same scheme could be safely applied to the studies concerning organic acid based PILs where the degree of ionization is known to be very high, and the neutral component can be assumed to be irrelevant or non-existent. Much more difficult is the study of AILs and AAILs where the degree of ionicity is not known a-priori and where the neutralization reactions may play an important role. In these cases, force field methods (even those with polarization) can easily fail, and more sophisticated approaches (typically AIMD) are needed in order to account for the change in the proton bonding patterns. The same issues affect also the studies of AAILs where a secondary proton may give rise to further acid/base equilibria leading to zwitterionic species. In this regard, the development of high-performance semiempirical methods such as DFTB can be a crucial factor in advancing the possibilities offered by numerical simulations. 


\section{Conflicts of interest}

There are no conflicts to declare.

\section{Notes and references}

1 J. W. Park, R. Al-Saadon, M. K. MacLeod, T. Shiozaki and B. Vlaisavljevich, Chem. Rev., 2020, 120, 5878-5909.

2 R. J. Bartlett and M. Musiał, Rev. Mod. Phys., 2007, 79, 291352.

3 A. D. Becke, J. Chem. Phys., 2014, 140, 18 A301.

4 S. Kozuch, D. Gruzman and J. M. L. Martin, J. Phys. Chem. C, 2010, 114, 20801-20808.

5 J. Tomasi, B. Mennucci and R. Cammi, Chem. Rev., 2005, 105, 2999-3094.

6 B. Mennucci, Wiley Interdiscip. Rev. Comput. Mol. Sci., 2012, 2, 386-404.

7 M. H. V. V. Regenmortel, EMBO Rep., 2004, 5, 1016-1020.

8 P. L. Luisi, Found. Chem., 2002, 4, 183-200.

9 B. K. Kendrick, J. Chem. Phys., 2018, 148, 044116.

10 T. E. Markland and M. Ceriotti, Nat. Rev. Chem., 2018, 2, 0109.

11 L. Monticelli and D. P. Tieleman, in Biomolecular Simulations, eds. L. Monticelli and E. Salonen, Humana Press, Totowa, NJ, 2013, vol. 924, pp. 197-213.

12 M. E. Tuckerman, J. Phys. Condens. Matter, 2002, 14, R1297R1355.

13 J. Hermans, B. Leimkuhler, S. Reich, A. E. Mark, P. Deuflhard and R. D. Skeel, Computational Molecular Dynamics: Challenges, Methods, Ideas: Proceedings of the 2nd International Symposium on Algorithms for Macromolecular Modelling, Berlin, May 21-24, 1997, .

14 R. B. Gerber, D. Shemesh, M. E. Varner, J. Kalinowski and B. Hirshberg, Phys Chem Chem Phys, 2014, 16, 9760-9775.

15 H. Nishizawa, Y. Nishimura, M. Kobayashi, S. Irle and H. Nakai, J. Comput. Chem., 2016, 37, 1983-1992.

16 D. R. MacFarlane and K. R. Seddon, Aust. J. Chem., 2007, 60, 3.

17 R. Hayes, G. G. Warr and R. Atkin, Chem. Rev., 2015, 115, 6357-6426.

18 H. L. Chum, V. R. Koch, L. L. Miller and R. A. Osteryoung, J. Am. Chem. Soc., 1975, 97, 3264-3265.

19 P. J. Scammells, J. L. Scott and R. D. Singer, Aust. J. Chem., 2005, 58, 155

20 X. Wang, C. A. Ohlin, Q. Lu, Z. Fei, J. Hu and P. J. Dyson, Green Chem., 2007, 9, 1191.

21 D. Zhao, Y. Liao and Z. Zhang, CLEAN - Soil Air Water, 2007, 35 42-48.

22 M. Petkovic, K. R. Seddon, L. P. N. Rebelo and C. Silva Pereira, Chem Soc Rev, 2011, 40, 1383-1403.

23 S. P. F. Costa, A. M. O. Azevedo, P. C. A. G. Pinto and M. L. M. F. S. Saraiva, ChemSusChem, 2017, 10, 2321-2347.

24 P. Kumari, V. V. S. Pillai and A. Benedetto, Biophys. Rev., 2020, 12, 1187-1215.

25 A. P. Abbott, D. Boothby, G. Capper, D. L. Davies and R. K. Rasheed, J. Am. Chem. Soc., 2004, 126, 9142-9147.

26 Y. Fukaya, Y. lizuka, K. Sekikawa and H. Ohno, Green Chem., 2007, 9, 1155.

27 J. Hulsbosch, D. E. De Vos, K. Binnemans and R. Ameloot, ACS Sustain. Chem. Eng., 2016, 4, 2917-2931.

28 J.-C. Plaquevent, J. Levillain, F. Guillen, C. Malhiac and A.-C. Gaumont, Chem. Rev., 2008, 108, 5035-5060.

29 J. M. Gomes, S. S. Silva and R. L. Reis, Chem. Soc. Rev., 2019, 48, 4317-4335.
30 S. K. Zandu, H. Chopra and I. Singh, Curr. Drug Res. Rev., 2020, 12, 26-41.

31 R. Moshikur, R. Chowdhury, M. Moniruzzaman and M. Goto, Green Chem., 2020, 22, 8116-8139.

32 V. B. Saptal and B. M. Bhanage, ChemSusChem, 2017, 10, 1145-1151.

33 X. Lu, G. Burrell, F. Separovic and C. Zhao, J. Phys. Chem. B, 2012, 116, 9160-9170.

34 T. L. Greaves and C. J. Drummond, Chem Soc Rev, 2013, 42, 1096-1120.

35 E. C. Wijaya, T. L. Greaves and C. J. Drummond, Faraday Discuss., 2013, 167, 191.

36 Y.-L. Wang, B. Li, S. Sarman, F. Mocci, Z.-Y. Lu, J. Yuan, A. Laaksonen and M. D. Fayer, Chem. Rev., 2020, 120, 57985877.

37 Z. Lei, B. Chen, Y.-M. Koo and D. R. MacFarlane, Chem. Rev., 2017, 117, 6633-6635.

38 P. D’Angelo, A. Zitolo, V. Migliorati, E. Bodo, G. Aquilanti, J. L. Hazemann, D. Testemale, G. Mancini and R. Caminiti, J. Chem. Phys., 2011, 135, 074505.

39 E. Bodo, L. Gontrani, A. Triolo and R. Caminiti, J. Phys. Chem. Lett., 2010, 1, 1095-1100.

40 A. Triolo, O. Russina, H.-J. Bleif and E. Di Cola, J. Phys. Chem. B, 2007, 111, 4641-4644.

41 P. A. Hunt, C. R. Ashworth and R. P. Matthews, Chem. Soc. Rev., 2015, 44, 1257-1288.

42 A. Knorr and R. Ludwig, Sci. Rep., 2015, 5, 17505.

43 A. Le Donne, H. Adenusi, F. Porcelli and E. Bodo, ACS Omega, 2018, 3, 10589-10600.

44 K. Dong, X. Liu, H. Dong, X. Zhang and S. Zhang, Chem. Rev., 2017, 117, 6636-6695.

45 L. Gontrani, Biophys. Rev., 2018, 10, 873-880.

46 A. Le Donne and E. Bodo, Biophys. Rev., , DOI:10.1007/s12551021-00782-0.

47 H. Ohno, K. Fukumoto and J. Kagimoto, in Ionic Liquids IV, eds. J. F. Brennecke, R. D. Rogers and K. R. Seddon, American Chemical Society, Washington, DC, 2007, vol. 975, pp. 351361

48 P. Moriel, E. J. García-Suárez, M. Martínez, A. B. García, M. A. Montes-Morán, V. Calvino-Casilda and M. A. Bañares, Tetrahedron Lett., 2010, 51, 4877-4881.

49 Q.-P. Liu, X.-D. Hou, N. Li and M.-H. Zong, Green Chem, 2012, 14, 304-307.

50 C. Jiang, W. Li, J. Nian, W. Lou and X. Wang, Friction, 2018, 6, 208-218.

51 S. Zhang, L. Ma, P. Wen, X. Ye, R. Dong, W. Sun, M. Fan, D. Yang, F. Zhou and W. Liu, Tribol. Int., 2018, 121, 435-441.

52 B. E. Gurkan, J. C. de la Fuente, E. M. Mindrup, L. E. Ficke, B. F. Goodrich, E. A. Price, W. F. Schneider and J. F. Brennecke, J. Am. Chem. Soc., 2010, 132, 2116-2117.

53 A.-H. Liu, R. Ma, C. Song, Z.-Z. Yang, A. Yu, Y. Cai, L.-N. He, Y.-N. Zhao, B. Yu and Q.-W. Song, Angew. Chem. Int. Ed., 2012, 51, 11306-11310.

54 M. Pan, Y. Zhao, X. Zeng and J. Zou, Energy Fuels, 2018, 32, 6130-6135.

55 X. Y. Luo, X. Fan, G. L. Shi, H. R. Li and C. M. Wang, J. Phys. Chem. B, 2016, 120, 2807-2813.

56 S. Onofri, H. Adenusi, A. Le Donne and E. Bodo, ChemistryOpen, 2020, 9, 1153-1160.

57 M. Guncheva, P. Ossowicz, E. Janus, S. Todinova and D. Yancheva, J. Mol. Liq., 2019, 283, 257-262. 
58 C. P. Song, R. N. Ramanan, R. Vijayaraghavan, D. R. MacFarlane, E.-S. Chan and C.-W. Ooi, ACS Sustain. Chem. Eng., 2015, 3, 3291-3298.

59 C. P. Song, R. N. Ramanan, R. Vijayaraghavan, D. R. MacFarlane, E.-S. Chan, J. A. P. Coutinho, L. Fernandez and C.W. Ooi, J. Chem. Thermodyn., 2017, 115, 191-201.

60 R. Wang, Y. Chang, Z. Tan and F. Li, J. Mol. Liq., 2016, 222, 836844.

61 M. T. Zafarani-Moattar, H. Shekaari and P. Jafari, J. Chem. Thermodyn., 2019, 130, 17-32.

62 X.-D. Hou, N. Li and M.-H. Zong, ACS Sustain. Chem. Eng., 2013, $1,519-526$

63 R. Wang, Y. Chang, Z. Tan and F. Li, Sep. Sci. Technol., 2016, 51, 1093-1102.

64 T. Q. To, K. Procter, B. A. Simmons, S. Subashchandrabose and R. Atkin, Faraday Discuss., 2018, 206, 93-112.

65 L. del Olmo, I. Lage-Estebanez, R. López and J. M. García de la Vega, J. Phys. Chem. B, 2016, 120, 10327-10335.

66 A. Benedetto, E. Bodo, L. Gontrani, P. Ballone and R. Caminiti, J. Phys. Chem. B, 2014, 118, 2471-2486.

67 D.-J. Tao, Z. Cheng, F.-F. Chen, Z.-M. Li, N. Hu and X.-S. Chen, J. Chem. Eng. Data, 2013, 58, 1542-1548.

68 M. Campetella, S. De Santis, R. Caminiti, P. Ballirano, C. Sadun, L. Tanzi and L. Gontrani, RSC Adv., 2015, 5, 50938-50941.

69 M. Campetella, D. C. Martino, E. Scarpellini and L. Gontrani, Chem. Phys. Lett., 2016, 660, 99-101.

70 O. Russina, S. De Santis and L. Gontrani, RSC Adv., 2016, 6, 34737-34743.

71 Y. Wang, S. Feng and G. A. Voth, J. Chem. Theory Comput., 2009, 5, 1091-1098.

72 Y. Wang, W. Jiang, T. Yan and G. A. Voth, Acc. Chem. Res. 2007, 40, 1193-1199.

73 J. Forsman, C. E. Woodward and M. Trulsson, J. Phys. Chem. B, 2011, 115, 4606-4612.

74 E. I. Izgorodina, J. Rigby and D. R. MacFarlane, Chem Commun, 2012, 48, 1493-1495.

75 B. Zhang, A. C. T. van Duin and J. K. Johnson, J. Phys. Chem. B 2014, 118, 12008-12016.

76 J. A. Conrad, S. Kim and M. S. Gordon, Phys. Chem. Chem. Phys., 2019, 21, 16878-16888.

77 T. Y. Nikolaienko and L. A. Bulavin, Int. J. Quantum Chem. 2019, 119, e25798.

78 E. Bodo, S. Mangialardo, F. Ramondo, F. Ceccacci and P. Postorino, J. Phys. Chem. B, 2012, 116, 13878-13888.

79 L. Tanzi, P. Benassi, M. Nardone and F. Ramondo, J. Phys. Chem. A, 2014, 118, 12229-12240.

80 Z. L. Seeger and E. I. Izgorodina, J. Chem. Theory Comput., 2020, 16, 6735-6753.

81 V. H. Paschoal, L. F. O. Faria and M. C. C. Ribeiro, Chem. Rev., 2017, 117, 7053-7112.

82 F. Dommert, K. Wendler, R. Berger, L. Delle Site and C. Holm, ChemPhysChem, 2012, 13, 1625-1637.

83 M. Salanne, Phys. Chem. Chem. Phys., 2015, 17, 14270-14279.

84 T. Köddermann, D. Paschek and R. Ludwig, ChemPhysChem, 2007, 8, 2464-2470.

85 E. J. Maginn, J. Phys. Condens. Matter, 2009, 21, 373101.

86 Y. Wang and G. A. Voth, J. Am. Chem. Soc., 2005, 127, 1219212193.

87 C. S. Santos, H. V. R. Annapureddy, N. S. Murthy, H. K. Kashyap, E. W. Castner and C. J. Margulis, J. Chem. Phys., 2011, 134, 064501.
88 A. Triolo, O. Russina, B. Fazio, R. Triolo and E. Di Cola, Chem. Phys. Lett., 2008, 457, 362-365.

89 H. V. R. Annapureddy, H. K. Kashyap, P. M. De Biase and C. J. Margulis, J. Phys. Chem. B, 2010, 114, 16838-16846.

90 C. Schröder, Phys. Chem. Chem. Phys., 2012, 14, 3089.

91 D. Bedrov, J.-P. Piquemal, O. Borodin, A. D. Mackerell, B. Roux and C. Schröder, Chem. Rev., 2019, 119, 7940-7995.

92 O. Hollóczki, F. Malberg, T. Welton and B. Kirchner, Phys Chem Chem Phys, 2014, 16, 16880-16890.

93 M. Brüssel, M. Brehm, A. S. Pensado, F. Malberg, M. Ramzan, A. Stark and B. Kirchner, Phys. Chem. Chem. Phys., 2012, 14, 13204.

94 H. Yu and W. F. van Gunsteren, Comput. Phys. Commun., 2005, 172, 69-85.

95 T. Yan, C. J. Burnham, M. G. Del Pópolo and G. A. Voth, J. Phys. Chem. B, 2004, 108, 11877-11881.

96 J. S. Tse, Annu. Rev. Phys. Chem., 2002, 53, 249-290.

97 P. J. Stephens, F. J. Devlin, C. F. Chabalowski and M. J. Frisch, J. Phys. Chem., 1994, 98, 11623-11627.

98 P. L. Silvestrelli, N. Marzari, D. Vanderbilt and M. Parrinello, Solid State Commun., 1998, 107, 7-11.

99 A. Le Donne and E. Bodo, J. Mol. Liq., 2018, 249, 1075-1082.

100 F. Malberg, M. Brehm, O. Hollóczki, A. S. Pensado and B. Kirchner, Phys. Chem. Chem. Phys., 2013, 15, 18424.

101 M. Brehm and D. Sebastiani, J. Chem. Phys., 2018, 148, 193802.

102 E. Roos and M. Brehm, Phys. Chem. Chem. Phys., 2021, 23, 1242-1253.

103 B. Doherty, X. Zhong and O. Acevedo, J. Phys. Chem. B, 2018, 122, 2962-2974.

104 G. Seifert, D. Porezag and Th. Frauenheim, Int. J. Quantum Chem., 1996, 58, 185-192.

105 M. Elstner, D. Porezag, G. Jungnickel, J. Elsner, M. Haugk, Th. Frauenheim, S. Suhai and G. Seifert, Phys. Rev. B, 1998, 58, 7260-7268.

106 M. Gaus, Q. Cui and M. Elstner, J. Chem. Theory Comput., 2011, 7, 931-948.

107 M. Gaus, X. Lu, M. Elstner and Q. Cui, J. Chem. Theory Comput., 2014, 10, 1518-1537.

108 M. Elstner, Theor. Chem. Acc., 2006, 116, 316-325.

109 M. A. Addicoat, R. Stefanovic, G. B. Webber, R. Atkin and A. J. Page, J. Chem. Theory Comput., 2014, 10, 4633-4643.

110 P. Goyal, H.-J. Qian, S. Irle, X. Lu, D. Roston, T. Mori, M. Elstner and Q. Cui, J. Phys. Chem. B, 2014, 118, 11007-11027.

111 J.-P. Belieres and C. A. Angell, J. Phys. Chem. B, 2007, 111, 4926-4937.

112 M. Yoshizawa, W. Xu and C. A. Angell, J. Am. Chem. Soc., 2003, 125, 15411-15419.

113 M. S. Miran, H. Kinoshita, T. Yasuda, Md. A. B. H. Susan and M. Watanabe, Phys. Chem. Chem. Phys., 2012, 14, 5178-5186.

114 P. Berton, S. P. Kelley, H. Wang and R. D. Rogers, J. Mol. Liq., 2018, 269, 126-131.

115 E. Bodo, Molecules, 2020, 25, 1432.

116 Y. Lv, Y. Guo, X. Luo and H. Li, Sci. China Chem., 2012, 55, 1688-1694.

117 J. Stoimenovski, E. I. Izgorodina and D. R. MacFarlane, Phys. Chem. Chem. Phys., 2010, 12, 10341.

118 M. S. Miran, M. Hoque, T. Yasuda, S. Tsuzuki, K. Ueno and M. Watanabe, Phys. Chem. Chem. Phys., 2019, 21, 418-426.

119 M. S. Miran, T. Yasuda, M. A. B. H. Susan, K. Dokko and M. Watanabe, ECS Trans., 2013, 50, 285-291. 
120 S. K. Davidowski, F. Thompson, W. Huang, M. Hasani, S. A. Amin, C. A. Angell and J. L. Yarger, J. Phys. Chem. B, 2016, 120, 4279-4285.

121 K. Chen, Y. Wang, J. Yao and H. Li, J. Phys. Chem. B, 2018, 122, 309-315.

122 X. Sun, S. Liu, A. Khan, C. Zhao, C. Yan and T. Mu, New J Chem, 2014, 38, 3449-3456.

123 J. E. S. J. Reid, C. E. S. Bernardes, F. Agapito, F. Martins, S. Shimizu, M. E. Minas da Piedade and A. J. Walker, Phys Chem Chem Phys, 2017, 19, 28133-28138.

124 A. T. Nasrabadi and L. D. Gelb, J. Phys. Chem. B, 2018, 122, 5961-5971.

125 E. Bodo, M. Bonomo and A. Mariani, J. Phys. Chem. B, 2021, 125, 2781-2792.

126 I. V. Fedorova and L. P. Safonova, J. Phys. Chem. A, 2019, 123, 293-300.

127 I. V. Fedorova and L. P. Safonova, J. Phys. Chem. A, 2018, 122, 5878-5885.

128 H. Adenusi, A. Le Donne, F. Porcelli and E. Bodo, J. Phys. Chem. B, 2020, 124, 1955-1964.

129 A. Le Donne, H. Adenusi, F. Porcelli and E. Bodo, J. Phys. Chem. $B, 2019,123,5568-5576$.

130 M. Campetella, E. Bodo, R. Caminiti, A. Martino, F. D’Apuzzo, S. Lupi and L. Gontrani, J. Chem. Phys., 2015, 142, 234502.

131 M. Campetella, A. Le Donne, M. Daniele, L. Gontrani, S. Lupi, E. Bodo and F. Leonelli, J. Phys. Chem. B, 2018, 122, 2635-2645.

132 T. Niemann, D. Zaitsau, A. Strate, A. Villinger and R. Ludwig, Sci. Rep., 2018, 8, 14753.

133 T. Niemann, J. Neumann, P. Stange, S. Gärtner, T. G. A. Youngs, D. Paschek, G. G. Warr, R. Atkin and R. Ludwig, Angew. Chem. Int. Ed., 2019, 58, 12887-12892.

134 T. Niemann, D. H. Zaitsau, A. Strate, P. Stange and R. Ludwig, Phys. Chem. Chem. Phys., 2020, 22, 2763-2774.

135 A. E. Khudozhitkov, J. Neumann, T. Niemann, D. Zaitsau, P. Stange, D. Paschek, A. G. Stepanov, D. I. Kolokolov and R. Ludwig, Angew. Chem. Int. Ed., 2019, 58, 17863-17871.

136 M. V. Fedotova, S. E. Kruchinin and G. N. Chuev, J. Mol. Liq., 2019, 296, 112081.

137 S. Miao, J. Wood, H. J. Jiang, S. Imberti, R. Atkin and G. Warr, J. Mol. Liq., 2020, 319, 114327.

138 S. Miao, R. Atkin and G. G. Warr, Phys. Chem. Chem. Phys., 2020, 22, 3490-3498.

139 M. Campetella, M. Montagna, L. Gontrani, E. Scarpellini and E. Bodo, Phys. Chem. Chem. Phys., 2017, 19, 11869-11880.

140 H. Adenusi, A. Le Donne, F. Porcelli and E. Bodo, J. Phys. Chem. $B, 2020,124,1955-1964$. 